IdeAs

Idées d'Amériques

16 | 2020

Les marges créatrices : intellectuel.le.s afrodescendant.e.s et indigènes auX Amériques, XIX-XXe siècle

\title{
America first bis repetita ou « aux côtés des alliés et des amis »?
}

Isabelle Vagnoux

\section{OpenEdition \\ Journals}

Édition électronique

URL : http://journals.openedition.org/ideas/9652

DOI : 10.4000/ideas.9652

ISSN : $1950-5701$

Éditeur

Institut des Amériques

\section{Référence électronique}

Isabelle Vagnoux, «America first bis repetita ou « aux côtés des alliés et des amis »? », IdeAs [En ligne], 16 | 2020, mis en ligne le 01 octobre 2020, consulté le 18 octobre 2020. URL : http:// journals.openedition.org/ideas/9652 ; DOI : https://doi.org/10.4000/ideas.9652

Ce document a été généré automatiquement le 18 octobre 2020

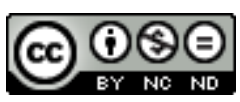

IdeAs - Idées d'Amériques est mis à disposition selon les termes de la licence Creative Commons Attribution - Pas d'Utilisation Commerciale - Pas de Modification 4.0 International. 


\title{
America first bis repetita ou « aux côtés des alliés et des amis »?
}

\author{
Isabelle Vagnoux
}

$129 \%$... rarement le monde a montré aussi peu de confiance envers le président américain ${ }^{1}$. Cependant, l'image des Etats-Unis résiste assez bien avec $54 \%$ d'opinions favorables. A l'évidence, même s'il apparaît bien soutenu par les électeurs de la droite populiste partout dans le monde, voire plébiscité en Israël (71\%), Donald Trump et ses choix stratégiques souffrent d'un déficit de popularité qui n'a pas renforcé la position internationale des Etats-Unis. Comment regagner une influence positive sur la communauté internationale? Les élections de 2020 peuvent-elles impulser une nouvelle donne?

2 Pour Joe Biden comme pour Donald Trump, une chose est certaine : ils entrent dans l'ère des "inconnues inconnues" (unknown unknowns), selon la formule de Donald Rumsfeld en 2002. En outre, plus que jamais, à l'heure de la pandémie, le paramètre intérieur s'invite dans la définition même de la politique étrangère, un véritable défi après des décennies dominées par le credo bipartisan de la mondialisation. En bref, l'heure est à la protection des intérêts « de Detroit plutôt que de Davos $»^{2}$. Dans le même temps, la Chine poursuit son ascension à un rythme soutenu. A l'évidence, Washington ne peut plus prétendre assurer seul le leadership d'un ordre mondial multipolaire, en pleine mutation et au bord d'un bras de fer entre deux superpuissances. A l'aube de cette période ponctuée d'incertitudes, l'élection présidentielle de 2020 revêt une importance particulière et nous nous proposons ici d'analyser à grands traits les principales orientations stratégiques des deux candidats en matière de politique étrangère.

\section{Les orientations générales}

3 L'absence de programme du Parti républicain lors de la convention de 2020 - fait sans précédent -, le simple renvoi au programme de $2016^{3}$, tout comme le discours de fin de convention de Donald Trump ou son document de campagne ${ }^{4}$ ne permettent pas de 
percevoir de nouveaux objectifs de politique étrangère en cas de réélection. Quelques brèves résolutions réaffirment le programme America first et le nationalisme afférent, confirment le soutien absolu à la souveraineté américaine et à une défense forte, à des politiques économiques créatrices d'emplois et de plus grande richesse pour les Américains, et à la « défense des droits de l'homme aux Etats-Unis comme partout dans le monde» $(s i c)^{5}$. Toute la campagne républicaine s'appuie d'ailleurs sur le bilan présidentiel et ses "promesses tenues ». Le libre-échange, clé de voûte de la politique américaine depuis des décennies? Au nom de la défense des intérêts des producteurs et des ouvriers américains, il est attaqué, avec des droits de douane frappant aussi bien les importations chinoises qu'européennes ou canadiennes; l'accord de libre-échange emblématique, l'ALENA, est renégocié avec le Canada et le Mexique, et perd son nom régional au profit de l'imprononçable USMCA. Les organisations internationales? La Maison-Blanche s'en défie et s'en retire (UNESCO, Organisation mondiale de la Santé). Les accords internationaux? Elle s'en retire également, détricotant tous les efforts multilatéraux auxquels l'Administration Obama avait adhéré (accord sur le nucléaire iranien; accords de Paris sur le climat; Partenariat transpacifique) ou plus anciens ("Ciel ouvert », sur la surveillance aérienne). Les alliances historiques ? A part Israël, qui voit sa "relation spéciale" renforcée, elles sont mises à mal. L'aide au développement? Victime de coupes drastiques cependant endiguées par le Congrès. La diplomatie? Délaissée au profit des confrontations (Corée du Nord, Iran, Chine) et d'une image martiale de l'Amérique, paradoxalement sans nouvelle intervention militaire à ce jour. Les relations internationales en soi ne revêtent pas grande importance pour Donald Trump, sauf pour glorifier la grandeur de l'Amérique ou pour arracher des concessions dans une négociation toujours bilatérale, et sont perçues essentiellement en lien avec l'électorat américain. Sans doute continuera-t-il sur le même mode transactionnel et impulsif et avec les mêmes objectifs. Nonobstant sa personnalité, il n'est toutefois pas exclu que, débarrassé du paramètre électoraliste après une seconde élection, il revienne vers une ligne plus conventionnelle.

Côté démocrate, dans un numéro d'équilibriste qui consiste à rallier électeurs de l'aile gauche, centristes et républicains modérés, on s'attache à prendre le contrepied de la méthode Trump. Ils entendent rassembler les Américains, rassurer le reste du monde, soutenir les démocraties (contrastant avec le penchant de Trump pour les autocrates, ce qui permet indirectement de prendre ses distances avec des alliés encombrants telles l'Arabie saoudite ou la Turquie), "sauver » la politique étrangère du pays ${ }^{6}$ en montrant l'exemple d'une Amérique multiculturelle, en redonnant tout son pouvoir à la diplomatie, délaissée au profit d'une «surmilitarisation $»^{7}$, en travaillant en concertation avec les autres nations. Joe Biden et les démocrates partent du principe qu'aucune nation ne peut régler seule les défis sanitaires, sécuritaires et environnementaux de ce siècle, et qu'il convient d'éviter un duel entre grandes puissances, susceptible d'aboutir à une nouvelle guerre froide. Ils prônent donc un retour au multilatéralisme et à la coopération internationale. Coopération mais... sous la houlette de Washington qui doit " renouveler, réinventer le leadership américain ${ }^{8}$, à la tête d'une vaste alliance des démocraties qu'il entend inspirer et guider dans un sens qui lui serait favorable ( Il incombe aux Etats-Unis de montrer le chemin. Aucune autre nation n'a cette capacité $\left.»^{9}\right)$, pour écrire les règles et les normes du monde de demain, comme l'équipe Truman l'avait fait dès la fin de la Seconde Guerre mondiale, excluant ainsi toute troisième voie, notamment européenne. 
5 Un des atouts de Joe Biden réside dans sa parfaite connaissance des dossiers de politique étrangère ou des questions "intermestiques" (à la fois internationales et intérieures, telles que le commerce ou l'immigration), après huit années passées aux côtés de Barack Obama en tant que vice-président, et trente-six années comme sénateur au Congrès des Etats-Unis, dont quatre en tant que président de la très influente commission des Affaires étrangères, même s'il ne semble pas avoir initié de réforme majeure. Le cas échéant, sa longue expérience au Congrès pourra lui permettre, à l'instar d'un Lyndon B. Johnson, de solliciter directement les sénateurs les plus à même de soutenir ses projets.

\section{Les enjeux régionaux}

6 Trois pays s'invitent dans la campagne électorale américaine : la Russie, la Chine et Israël. La première est soupçonnée par les services de renseignements depuis 2016 de chercher à s'immiscer dans les résultats de l'élection; elle sera par ailleurs très rapidement sur le devant de la scène diplomatique avec la fin du traité sur la réduction des armements stratégiques (New START) en février 2021 et la question des droits de l'homme. Sanctions en cas de nouvelles accusations d'ingérence ? Retrait américain du traité ? Coopération? Négociation? Ce sera en tout cas l'un des premiers dossiers de la nouvelle Administration.

7 Accusée par Donald Trump d'avoir laissé répandre le coronavirus, la Chine se trouve quant à elle dans l'œil du cyclone trumpien, au cœur des préoccupations intermestiques et donc électoralistes, alors que la première partie de l'accord commercial, signé en janvier 2020, semblait avoir calmé des relations houleuses. Economiquement et financièrement interdépendants, concurrents en matière commerciale, les deux pays le sont également dans les domaines de l'intelligence artificielle, de la haute technologie et, de plus en plus, au plan militaire. Les violations des droits de l'homme, ainsi que le sort de Hong Kong, font l'objet, à Washington, de sanctions contre Pékin qui, par sa Loi de sécurité nationale, ne respecte pas l'accord signé en 1997 avec le Royaume-Uni. Historiquement soutenu par les milieux d'affaires et le Congrès américains, Taiwan sera-t-il le prochain sur la liste ? L'île se trouve au centre de la (re)définition du rôle des Etats-Unis dans la région.

8 Même si chacun des deux candidats accuse l'autre de s'être montré trop conciliant envers Pékin, ils partagent largement le même diagnostic sur l'expansionnisme chinois et la menace qu'il constitue pour le leadership américain et prônent une ligne sans concession. La différence réside dans la stratégie choisie : transactionnelle et parfois brutale pour Trump ; multilatéraliste pour Biden. Celui-ci entend renforcer la position de Washington en l'associant à ses alliés : liens resserrés, y compris au plan militaire, avec les alliés de l'Asie-Pacifique (Australie, Japon, Corée du Sud) afin de réaffirmer la position des Etats-Unis en tant que puissance Pacifique; coopération avec les "démocraties du monde " pour faire front commun contre les violations des lois commerciales et des droits de l'homme; concertation avec l'Europe pour gérer la relation avec la Chine.

9 Pour autant, le programme démocrate ne fait pas mention d'une réintégration des Etats-Unis au sein du Partenariat transpacifique, perçu par l'Administration Obama comme un moyen de faire pièce à la Chine, et dont Donald Trump était sorti dès 2017. La candidate à la vice-présidence, Kamala Harris, s'est d'ailleurs déclarée hostile à ce 
partenariat jugé contraire aux intérêts des Etats-Unis en termes d'emplois et de normes environnementales. Le partenariat «stratégique » avec l'Inde, grand rival de la Chine en Asie, fait également partie de cette politique d'encerclement. Développée sous George W. Bush en partie grâce à la diaspora indienne, la relation pourrait se voir renforcée par les origines indiennes de K. Harris, bien que celle-ci sache également se montrer critique envers Delhi. Enfin, la volonté de coopérer avec la Chine (et la Russie) demeure forte, notamment sur la pandémie, le changement climatique et la nonprolifération nucléaire, domaine dans lequel, sous George W. Bush, Pékin avait réussi à contenir la fièvre nucléaire de la Corée du Nord.

Quant à Israël, elle a fait une entrée fracassante dans la campagne lorsque l'Administration Trump a annoncé triomphalement en août 2020 un accord historique d'ouverture des relations commerciales et, à terme, diplomatiques, entre Israël et les Emirats arabes unis, et depuis avec Bahreïn. Premier accord de ce type entre l'Etat hébreu et un Etat de la Ligue arabe depuis 1994, prometteur d'accords semblables avec d'autres pays arabes partageant avec Jérusalem et Washington l'hostilité à l'égard de l'Iran, il s'agit là d'une victoire diplomatique pour l'administration Trump, et d'un bel atout au plan électoral puisque les chrétiens évangéliques américains comptent parmi les plus fervents défenseurs d'Israël, aux côtés du lobby pro-Israël (AIPAC). Déjà ravis du retrait des Etats-Unis de l'accord sur le nucléaire avec l'Iran et du transfert de l'ambassade américaine de Tel Aviv à Jérusalem en 2018, ces électeurs soutiendront sans nul doute Donald Trump pour un deuxième mandat. Inhabituelle, l'intervention du Secrétaire d'Etat, Mike Pompeo, depuis Jérusalem, lors de la convention républicaine, résume à elle seule le positionnement de D. Trump sur ce dossier.

11 Seule subsiste une ambiguïté sur la poursuite de l'occupation de la Cisjordanie, " suspendue », selon Jérusalem, mais non arrêtée. Or c'est bien la question épineuse des territoires occupés qui avait jusqu'alors empêché tout accord, les pays arabes et les démocrates tenant à une solution pour les Palestiniens. La peur de l'Iran aurait-elle mis la question palestinienne aux oubliettes? Prudent, le programme démocrate confirme le maintien de la capitale à Jérusalem (Joe Biden a toujours été un fervent défenseur d'Israël), mais promet des négociations en vue de la création de deux Etats et d'une solution pérenne à la question palestinienne. Ailleurs au Moyen-Orient, J. Biden met l'accent sur la fin de l'engagement massif dans des "guerres sans fin", le refus de participer, même indirectement, à tout changement de régime - rejoignant en cela $\mathrm{D}$. Trump -, la primauté donnée à la diplomatie, la poursuite de la lutte contre Daech, et le retour au sein de l'accord sur le nucléaire iranien, en concertation étroite avec les autres membres pour le renforcer.

12 Alors que D. Trump fait peu de cas de l'Europe (notamment occidentale), le programme démocrate souligne au contraire le caractère «crucial» de la coopération transatlantique, que ce soit pour soutenir l'OTAN, alliance militaire « exceptionnelle », ou pour œuvrer ensemble en faveur de la défense de la démocratie, sur les grands dossiers multilatéraux, ou à l'égard de la Russie, de la Chine ou de l'Iran. En bref, même si l'alliance atlantique s'est affaiblie tout au long de ce xxi siècle, même si, depuis Obama, les partenaires de l'OTAN sont sommés de contribuer davantage, les démocrates s'engagent à redonner vigueur à cette alliance historique perçue comme centrale sur l'échiquier mondial. L'attitude martiale de la Turquie (membre de l'OTAN) en Méditerranée orientale constituera l'un des premiers dossiers auquel le président américain devra s'intéresser. 

les pays des Amériques ( «somos una familia » disait Bush père en 1990), la coopération, le partenariat mais aussi la responsabilité partagée, alors que le "Sud " n'intéresse guère $\mathrm{D}$. Trump. Les démocrates proposent de remettre la diplomatie en selle dans la crise vénézuélienne et de réactiver la normalisation mise en place par Obama avec Cuba. Un des enjeux principaux pour la région, durement impactée par le COVID-19, est le dossier migratoire, qui ne la concerne pas exclusivement mais l'affecte fortement. Alors que D. Trump a pris des décisions brutales sur cette question, en phase avec son électorat, les démocrates évoquent l'essence même des Etats-Unis, "une nation d'immigrés ", selon la formule de J.F. Kennedy, et s'inscrivent dans une tradition américaine remontant aux premières heures de la nation. A la suite de B. Obama, ils reconnaissent que le système migratoire actuel «ne fonctionne pas » et entendent le « réinventer » dans le respect des valeurs américaines, et s'attaquer aux racines mêmes de l'émigration, travail de très, très longue haleine...

L'élection du 3 novembre 2020 n'est pas seulement présidentielle. Si l'hôte de la Maison-Blanche demeure seul maître en matière de politique étrangère, la coloration politique du Sénat et de la Chambre des Représentants a également un impact. En cas de "gouvernement partagé » (divided government), à savoir en cas de majorité au Congrès différente du parti du président, des équilibres, des compromis devront être trouvés, notamment pour les questions intermestiques. Comme il sied à tout président, les deux candidats partagent la même volonté de défendre avant tout les intérêts de leur pays, mais les enjeux dépassent largement le strict cadre américain : l'échiquier mondial et la gestion des relations internationales subiront à n'en point douter l'influence du résultat de ces élections tenues dans un climat sans précédent.

\section{NOTES}

1. Pew Research Center, «Trump Ratings Remain Low Around Globe », 8 janvier 2020, https:// www.pewresearch.org/global/2020/01/08/trump-ratings-remain-low-around-globe-whileviews-of-u-s-stay-mostly-favorable/, consulté le 27 août 2020.

2. Lawrence H. Summers, "How to Fix Globalization - For Detroit, not Davos », The American Interest, vol. 15, $\mathrm{n}^{\circ}$ 6, 22 mai 2020.

3. GOP, «Resolution Regarding the Republican Platform», https://prod-cdn-static.gop.com/ docs/Resolution_Platform_2020.pdf?

_ga=2.165306300.2055661719.1598124638-455285808.1584478680, consulté le 1 septembre 2020 .

4. Donald J. Trump, https://www.donaldjtrump.com/media/trump-campaign-announcespresident-trumps-2nd-term-agenda-fighting-for-you, consulté le 4 septembre 2020.

5. GOP, «Resolution in Support of America First Principles », https://prod-cdn-static.gop.com/ media/documents/RESOLUTION_IN_SUPPORT_OF_AMERICA_FIRST_PRINCIPLES.pdf?

_ga=2.173278179.1541020056.1598458103-337432649.1598458103, consulté le 1 septembre 2020.

6. Joe Biden, «Why America Must Lead Again: Rescuing U.S. Foreign Policy After Trump ", Foreign Affairs, mars-avril 2020, p. 64-76. 
7. Robert Gates, «The Overmilitarization of American Foreign Policy », Foreign Affairs, juil.-août, 2020, pp. 121-132.

8. Democratic Party Platform, 2020, in Gerhard Peters and John T. Woolley, The American Presidency Project, https://www.presidency.ucsb.edu/documents/2020-democratic-partyplatform, consulté le 4 septembre 2020.

9. Biden, art. cit.

\section{AUTEUR}

\section{ISABELLE VAGNOUX}

Isabelle Vagnoux, Aix Marseille Université, LERMA, UR 853, Aix-en-Provence, France 